\title{
Linking Experimental Solute Segregation Specificity in Nanocrystalline Alloys to Computational Predictions
}

\author{
Xuyang Zhou ${ }^{1}$ and Gregory B. Thompson ${ }^{1}$ \\ 1. The University of Alabama, Department of Metallurgical \& Materials Engineering, Tuscaloosa, AL \\ USA.
}

$\mathrm{Fe}(\mathrm{Cr})$ alloys are of strong technical interest because of their resistance to radiation effects, e.g. damage accumulation and swelling in future nuclear reactors [1, 2], as well as Cr's ability to retard Fe's oxidation. In each of these applications, Cr's placement within the grain boundaries is essential in achieving the desired properties. Nevertheless, this segregation is grain boundary specific and the ability to detect it requires high chemical sensitivity and high spatial resolution. In this paper, we discuss a cross-correlative microscopy technique to quantify $\mathrm{Cr}$ 's grain boundary specificity which has been used to verify and validate a computational model for solute segregation.

A series of $\mathrm{Fe}-\mathrm{XCr}$, where $\mathrm{X}=0-17$ at. \%, were sputtered as nanocrystalline alloys where upon its solute segregation tendencies were quantified using a cross-correlative precession electron diffraction (PED) - atom probe tomography (APT) technique [3, 4]. In order to link these experimental findings to computational models of the grain boundary types, one must know at least five of the grain boundary parameters. Three parameters can be obtained by the boundary misorientation whereas the other two are determined by the grain boundary inclination [5]. Using a recent technique reported by Kiss and Lábár [6], the inclination angle of the boundaries was quantified by PED of an atom probe tip prior to its field evaporation, Fig. 1(a)-(b). As one approaches the grain boundary, the diffraction patterns on either side of the boundary overlap and by weight averaging each pattern's intensity, the inclination angle is then measured albeit at the spatial resolution of the TEM probe $(\sim 1.5 \mathrm{~nm})$. Though, one disadvantage of this PED method for inclination determination is that it is based on a projection creating ambiguity to which side of the boundary is tilted. Here, this issue can be resolved by linking the curvature of the boundary directly from the 3D atom renderings (isosurfaces) to the PED scan. Thus, the cross-correlation with APT improved the fidelity of the PED reconstruction, and PED provided APT the necessary crystallographic information for the solute concentration specificity within the APT quantified boundary.

For the as-deposited Fe-8Cr film, specific grain boundary segregation was found, Fig. 1(c). The highest concentration being in the high angle grain boundaries followed by $\Sigma 3, \Sigma 9$, and $\Sigma 11$. The solute content within the $\Sigma 3$ boundaries was observed to be highly dependent on the inclination angle [4]. By quantifying the full character of the boundaries, the specific grain boundary was directly simulated using a hydride Monte Carlo - Molecular Dynamics method. The computational segregation trended well with the experimentally determined values, Fig. 1(c). In the high angle grain boundaries, the onset of $\mathrm{Cr}$ clustering was noted. With annealing during deposition, spinodal decomposition was observed in both low and high $\mathrm{Cr}$ content alloys suggesting that grain boundary clustering becomes less significant in the nanostructure evolution, Fig. 2. This resulted in the grain refinement. The detail links of how $\mathrm{Cr}$ content trends with boundary types and its effect on segregation (at specific boundaries and within the grain) will be discussed through the use of this PED-APT cross-correlative technique. 


\section{References:}

[1] Malerba, L. et al, Journal of Nuclear Materials 382 (2008), p. 112-125.

[2] Klueh, R. et al, Journal of Nuclear Materials 371 (2007), p. 37-52.

[3] Zhou, X. et al, Thin Solid Films 612 (2016), p. 29-35.

[4] Zhou, X. et al, Sci. Rep. 6 (2016), p. 34642.

[5] Pirgazi, H. et al, Journal of Applied Crystallography 48 (2015), p. 1672-1678.

[6] Kiss, A. K. et al, Microscopy and Microanalysis 22 (2016), p. 551-564.

[7] The authors gratefully recognize support from the Army Research Office, grant W911NF1310436, as well as the University of Alabama's Central Analytical Facility.
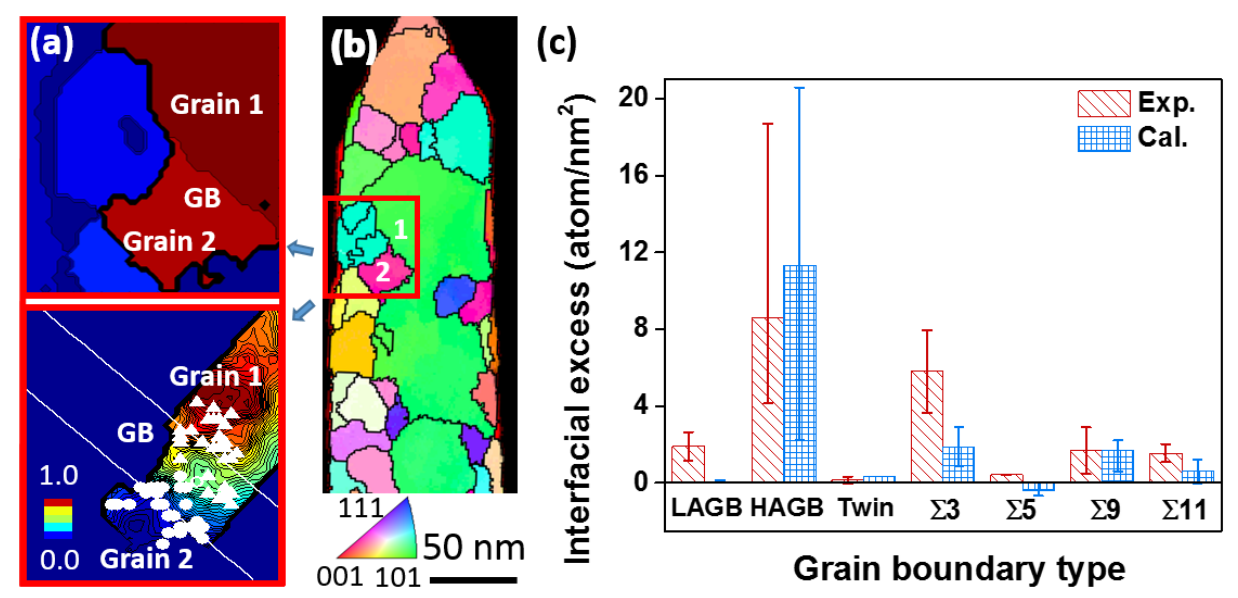

Figure 1. (a) Magnified image of a region of interest from a Fe-8Cr APT tip and the corresponding change in diffracted intensity (weight factor) of Grain 1 as it approaches the grain boundary projection, indicted by the white line. The white marked features (triangles/spheres) represent the weight factor locations used to fit the projected boundary line. (b) Orientation map of the Fe-8Cr APT tip. (c) Experimental and simulated interfacial excesses varying with grain boundary types. Error bars represent upper and lower limits. Cal. (calculated) refers to the simulated boundaries. Figure adapted from author's reference [4].

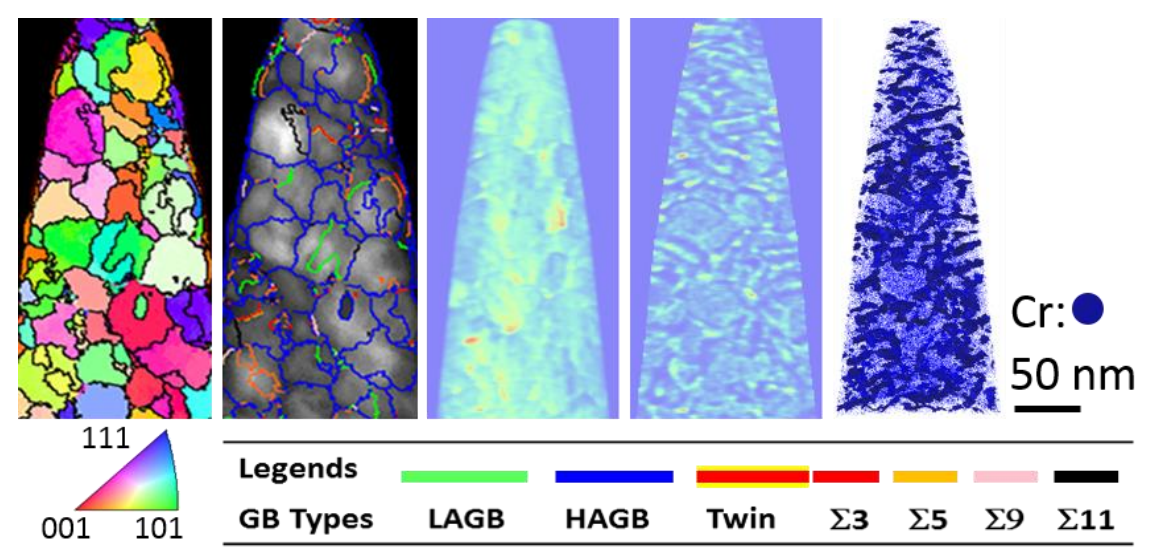

Figure 2. (a) Microstructure and composition mapping of $\mathrm{Fe}-4 \mathrm{Cr}$ at $400^{\circ} \mathrm{C}$. From left to right: the PED orientation map of the atom probe tip, CSL boundary line map laid over the image quality map of the tip, 2D density profile map, 2D composition profile map, atom map image with an Cr isodensity surface. 